Revista Arbitrada Interdisciplinaria KOINONIA

Año VI. Vol VI. N³. Edición Especial: Educación II. 2021

Hecho el depósito de Ley: FA2016000010 ISSN: 2542-3088

FUNDACIÓN KOINONIA (F.K). Santa Ana de Coro. Venezuela.

Christian Gerardo Quinteros-Pallarozo; Nancy Marcela Cárdenas-Cordero

http://dx.doi.org/10.35381/r.k.v6i3.1306

\title{
Aula invertida y juego de roles: Implementación en el bachillerato técnico agropecuario
}

Flipped classroom and roll play: Implementation in the agricultural technical baccalaureate

\author{
Christian Gerardo Quinteros-Pallarozo \\ christian.quinteros.01@est.ucacue.edu.ec \\ Universidad Católica de Cuenca, Azogues \\ Ecuador \\ https://orcid.org/0000-0003-2377-8985 \\ Nancy Marcela Cárdenas-Cordero \\ ncardenasc@ucacue.edu.ec \\ Universidad Católica de Cuenca, Azogues \\ Ecuador \\ https://orcid.org/0000-0002-6250-6504
}

Recepción: 10 de abril 2021

Revisado: 05 de mayo 2021

Aprobación: 30 de junio 2021

Publicación: 15 de julio 2021 


\title{
RESUMEN
}

La presente investigación estuvo encaminada a implementar el aula invertida y juego de roles en el Bachillerato Técnico de Producción Agropecuaria de la Unidad Educativa "José Benigno Iglesias" de la Provincia de Cañar, Cantón Biblián, pues el poco interés que han mostrado los estudiantes por estas materias en los últimos años, ha generado que su rendimiento sea bajo y la adquisición de competencias que exige el mundo laboral no se vea fortalecido durante el proceso de enseñanza-aprendizaje. La investigación es de tipo descriptiva no experimental, posee un paradigma epistemológico cuantitativo y de cohorte transversal, con una muestra estratificada de estudiantes, donde se obtuvo como principal resultado que la combinación de estas dos metodologías, incrementa la participación y motivación en los estudiantes, esto se vuelve en un pilar muy importante para el desarrollo de las diferentes habilidades y destrezas necesarias para la resolución de problemas.

Descriptores: Estrategias educativas; aprendizaje active; autoaprendizaje. (Palabras tomadas del Tesauro UNESCO).

\begin{abstract}
This research was aimed at implementing the flipped classroom and role play in the Technical Baccalaureate of Agricultural Production of the Educational Unit "José Benigno Iglesias" of the Province of Cañar, Cantón Biblián, since the little interest that students have shown in these subjects in recent years, has caused their performance to be low and the acquisition of skills required by the world of work is not strengthened during the teaching-learning process. The research is of a descriptive, non-experimental type, it has a quantitative epistemological paradigm and a cross-sectional cohort, with a stratified sample of students, where it was obtained as the main result that the combination of these two methodologies increases participation and motivation in students, this it becomes a very important pillar for the development of the different abilities and skills necessary for problem solving.
\end{abstract}

Descriptors: Educational strategies; activity learning; self-instruction. (Words taken from the UNESCO Thesaurus). 
Revista Arbitrada Interdisciplinaria KOINONIA

Año VI. Vol VI. N³. Edición Especial: Educación II. 2021

Hecho el depósito de Ley: FA2016000010 ISSN: 2542-3088

FUNDACIÓN KOINONIA (F.K). Santa Ana de Coro. Venezuela.

\section{INTRODUCCIÓN}

Durante los últimos años se ha observado un creciente desinterés por parte de los estudiantes hacia ciertas figuras profesionales del bachillerato técnico y más específicamente hacia el de Producción Agropecuaria, esto ha generado que su rendimiento sea muy bajo. Por otra parte, investigaciones realizadas en otras Instituciones Educativas han demostrado que existe la misma problemática que se vive en la Unidad Educativa "José Benigno Iglesias", en cuanto al poco interés que los estudiantes tienen hacia esta carrera, produciendo una deserción o una demanda muy baja de la misma.

Así mismo, la utilización de metodologías activas en este Bachillerato Técnico ha sido muy poca o nula por parte de algunos docentes, generando otra problemática sobre la motivación que se puede formar en los estudiantes con los mismos. Adicionalmente, las metodologías empleadas hasta el día de hoy han sido las tradicionalistas, provocando que los estudiantes se conviertan en agentes pasivos del proceso de enseñanzaaprendizaje, sin lograr que ellos adquieran las habilidades y competencias necesarias que el bachillerato técnico así lo demanda.

Cabe recalcar que, los docentes enseñan desde su pizarrón, lo cual aleja la práctica de lo teórico, tal vez por un desconocimiento de estas en la didáctica empleada, a más de esto, el desconocimiento de tecnologías limita que se puedan implementar las metodologías activas que combinadas pueden generar un aprendizaje significativo, motivando a la reflexión, criticidad e investigación.

Como mencionan (Konopka et al. 2015), una metodología activa ayudará a los estudiantes a desarrollar una mejor comprensión del tema expuesto y de esta manera, generar habilidades y actitudes necesarias para un aprendizaje real, de esta forma dejar de lado actividades que se enfoquen solo en la transmisión de información promoviendo un aprendizaje superficial y no uno profundo.

Todo lo antes mencionado, implica que tanto los estudiantes como los docentes deben adaptarse a las diferentes situaciones, aprender a detectar problemas y responder con 
propuestas productivas viables, empleando sus conocimientos técnicos, mediante el uso de las tecnologías actuales que nos permiten tener una respuesta más adecuada a la problemática que puede surgir en el campo. Es así que, para evaluar si es o no factible la implementación de estas metodologías, hay que tomar en cuenta la diversidad de los estudiantes en un aula y buscar ambientes que favorezcan la experimentación y reflexión de los mismos mediante resultados que indicarán si estos favorecen o no el proceso de aprendizaje (Islas-Torres \& Carranza-Alcántar, 2020).

Por tanto, el principal objetivo de esta investigación es: implementar metodologías activas a través del aula invertida y juego de roles en el tercero de Bachillerato Técnico de Producción Agropecuaria en la Unidad Educativa “José Benigno Iglesias" período 2020 2021, para promover la motivación e interés en el aprendizaje de los estudiantes.

\section{Referencial teórico}

En los últimos años se ha evidenciado que el proceso de enseñanza-aprendizaje no ha variado en cuanto a las metodologías utilizadas por parte de los docentes, el desconocimiento de nuevas metodologías activas, tecnologías emergentes y las estrategias implementadas dentro del aula han generado que los estudiantes sean simples receptores de órdenes, mostrando claramente un sistema tradicionalista que provoca un claro desinterés en ellos. Es fundamental que el profesor conozca más de cerca a su estudiantado y la realidad en la que ellos se desenvuelven en el día a día. A más de esto, es necesario que sean llevados de forma crítica, reflexiva y flexible para promover el interés de las diferentes asignaturas (Rodríguez et al. 2017).

Por otra parte, para desarrollar habilidades metacognitivas en los estudiantes es necesario recoger cada una de sus necesidades y llevarlas al contexto real, abriendo un abanico de posibilidades más extensas e interesantes. También cabe mencionar que, hasta el día de hoy, la educación se ha centrado en parámetros de evaluación sumativa dejando de lado todo el proceso de formación que los estudiantes adquieren durante el 
proceso de aprendizaje, lo que impide que no se generen las competencias necesarias para afrontar la vida real (Muntaner-Guasp et al. 2020).

Como mencionan (Medina-Díaz \& Verdejo-Carrión, 2020), la aplicación de las diversas metodologías requiere reformular, proyectar y guiar la enseñanza de diferentes maneras, estas deben centrarse en técnicas e instrumentos de evaluación que vayan acorde a las situaciones que surgen en el ámbito profesional con estrategias que faciliten el logro de competencias por parte del alumnado ante una sociedad cambiante y que exige una innovación constante.

Para dejar de lado los métodos tradicionales donde las exposiciones o clases magistrales son su principal pilar y el conocimiento transmitido por los docentes no tiene mayor relevancia ya que, su aprendizaje es superficial, es indispensable incorporar nuevas metodologías, donde el conocimiento sea construido desde el protagonismo del estudiante, utilizando los principios fundamentales de este, puesto que, todas las técnicas son efectivas siempre y cuando sean aprovechadas cada uno de estas, no solo focalizadas en aspectos que lleven el nivel de exigencia mínima por parte de sus actores, sí no que, generen proactividad y autonomía (Silva \& Maturana, 2017).

Como es conocido, para lograr la construcción del conocimiento es indispensable partir de vivencias previas, de esta manera, se facilita la comprensión de los contenidos estudiados y se integran al alumno, lo que da pasó a que, las metodologías activas sean el camino conductor a superar los retos, solucionar conflictos y aplicar destrezas ante alguna situación adversa (Batistello, \& Cybis-Pereira, 2019).

\section{Aula Invertida}

Al ser una de las metodologías activas más utilizadas en los últimos tiempos y al ofrecer una gran variabilidad para los diferentes niveles, esta abre la oportunidad de desarrollar competencias en contextos que requieren de la aplicación de resolución de problemas, ya que, su intención es promover un aprendizaje real, puesto que, las actividades a las 
que se enfocan son: incentivar un proceso antes, durante y después de la clase. Estás se basan en tres principios:

1) el punto de vista cognitivo de Piaget, enfocado en la resolución de problemas, aprendizaje dinámico y colaborativo,

2) 2) la parte general de Vygotsky desde el desarrollo próximo o aprendizaje cooperativo, (3) las formas de aprendizaje experiencial de Kolb (HernándezSuárez et al. 2020).

Los grandes beneficios que ofrece esta metodología para los docentes son muy altos, esto se puede observar en las investigaciones realizadas por (Han \& Rokenes, 2020), quienes muestran un incremento en el rendimiento de las diferentes asignaturas estudiadas, para ello, evaluaron varios parámetros como: la motivación, actitud, entrega de contenido, entorno de aprendizaje centrado en el alumno, clases menos estresantes, lo que generó un positivismo, así como también la percepción y la confianza tuvo una mejora. Seguido de esto, podemos decir que, un trabajo que es incorporado dentro del aula, incentiva a un debate entre profesor-estudiante de temas que han sido previamente enviados por docentes en videos pregrabados, donde se puede encontrar una explicación de los temas a tratar en clase, lo que conlleva a estimular la curiosidad por saber que sigue después de lo expuesto (Castro et al. 2020).

Cabe señalar que, el aula invertida posibilita un aprendizaje a un ritmo propio y en ocaciones se obtiene una mayor profundización de los temas, para lograr los objetivos que el aula invertida nos ofrece es indispensable que el alumno eleve su grado de responsabilidad al asumir un proceso que le ayudará a construir un aprendizaje propio, enfocado a su contexto, esto da pie a que el docente pueda genarar recursos individualizados, lluvia de ideas, foros, tareas interactivas, cuestionamientos, análisis de problemas reales y juegos en línea, siempre orientadas a conseguir las metas planteadas de acuerdo a las necesidades detectadas dentro y fuera de clase, donde la guía del profesor será primordial (Vidal et al. 2016). 
Si bien es cierto que, se han realizado numerosas investigaciones del aula invertida como método innovador y que permite evaluar los procesos de rendimiento académico, proactividad de los estudiantes durante una clase, grado de responsabilidad y aumento en la motivación, los mismos han sido implementados solo en asignaturas ligadas a las ciencias sociales, naturales, matemáticas y computación, como lo muestran (EscuderoNahón \& Mercado-López, 2019), (Cantuña-Avila \& Cañar-Tapia, 2020) en sus investigaciones bibliográficas.

Lo anteriormente expuesto, nos hace suponer que, la implementación de esta ha sido nula en materias del bachillerato técnico, en especial en la figura profesional Producción Agropecuaria, así mismo, se convierte de vital importancia que se generen las competencias básicas necesarias mediante la participación eficaz del estudiante, contribuyendo en su aprendizaje y adquisición de nuevas conceptualizaciones en cuanto a su práctica, que le permite reorganizarse y descubrir nuevas ideas, de esta forma crear un reto cognitivo en ello.

Esto da paso a que se generé un interés marcado por querer seguir indagando en temáticas que le permitirán desarrollar las competencias necesarias que La Ley Orgánica de Educación Intercultural en art. 43, literal b dispone para los bachilleratos técnicos, para proveer "una formación complementaria que faculte el ingreso al campo laboral" (Ley Organica de Educación Intercultural LOEI) y los artículos 34, 35, 36 y 37 del Reglamento de la LOEl". Dichas competencias se las obtendrán mediante la aplicación del aula invertida junto a otras metodologías como el juego de roles.

\section{Juego de roles}

Si bien es cierto que, el poco interés que se genera en los estudiantes por ciertas materias, ha llevado a la busqueda insesante de nuevas metodologías que permitan acercarse de una forma más certera al desarrollo de sus competencias, y que, las mismas motiven a continuar con sus estudios, dejando de lado la parte memorística, la cual, no permite la mejora en su proceso de formación, ya sea por la perdida del interés paulatino 
Revista Arbitrada Interdisciplinaria KOINONIA

Año VI. Vol VI. N³. Edición Especial: Educación II. 2021

Hecho el depósito de Ley: FA2016000010

ISSN: 2542-3088

FUNDACIÓN KOINONIA (F.K). Santa Ana de Coro. Venezuela.

Christian Gerardo Quinteros-Pallarozo; Nancy Marcela Cárdenas-Cordero

de ciertas asignaturas en las que la práctica es mucho más importante que otras, o a su vez, porque el profesor no ha implementado una correcta didáctica dentro de las clases que imparte (Pons-García \& Sonsoles-de Soto-García, 2020).

Todo debe estar orientado a que se genere un aprendizaje significativo, por lo tanto las estrategias que se busquen debe lograr este objetivo principal. A más de ello, debemos acotar que, en el ámbito profesional se busca personas competentes acorde con las necesidades que cada profesión así lo requiere (Suárez-Cretton et al. 2020).

La metodología de juego de roles presenta la oportunidad de desarrollar habilidades para resolver casos que surgen en los diferentes ámbitos laborales, el diagnóstico de estos permite que se haga una reflexión profunda de la problemática surgida, involucra a cada alumno a trabajar cooperativamente en miras de llegar a las posibles soluciones de los casos, llevando a la práctica las teorías estudiadas previamente.

Muchas veces se trabaja desde un ámbito que se desapega de la realidad con ejemplos que son muy antiguos y han perdido su relevancia ante un mundo tan cambiante, estructuras poco convencionales y ortodoxas, es así que, se pretende dotar de las herramientas necesarias para que cada persona sea capaz de tomar desiciones cuando se encuentra ante otros pares (Gorbaneff, 2003). A más de esto, abre camino hacía un pensamiento profundo y reflexivo, lo que conlleva a desarrollar confianza al momento de su intervención y uso de sus aptitudes para solventar conflictos que otras personas viven en su medio (Suárez-Cretton et al. 2020).

El juego es muy transcendental durante la vida de una persona, ya que la experiencia vivida en la misma será positiva y recordada durante mucho tiempo, es mediante este aspecto que su utilización en la pedagogía permite que los estudiantes puedan comprender mejor una temática, además de divertirse durante el aprendizaje (GaeteQuezada, 2011). Por lo tanto, la información a investigar sobre la problemática debe ser accesible, coherente debatible, solventable y útil, acorde a la actualidad, lo que contribuye a obtener compentencias elementales para relacionarse con el universo físco o científico, a más de ello, la conexión con la naturaleza es donde se llevan a cabo un sin número de 
desafíos a ser resueltos y esto da paso a que se puedan implementar pedagogías enfocadas a proveer las herramientas necesarias que deben ser utilizadas en el momento propicio (España-Ramos et al. 2013).

Para el bachillearto técnico uno de los principales retos es la de fomar profesionales capacitados, dichas capacidades deben ser adquiridas durante su proceso formativo con metodologías que le permitan responder a las expectativas del mundo laboral, pero estas habilidades se obtienen con un trabajo coordinado entre estudinates y docentes que deben involucarse en lograr cumplir dichas expectativas.

Lamentablemente el poco interés por parte de los alumnos y el corto tiempo disponible para enlazar la parte teórica con la práctica, limita la capacidad reflexiva, crítica y resolutiva del campo agropecuario, pues se pretende enseñar a sembrar desde un pizarrón, lo que conlleva que el ciclo conductista se repita ya que, se cree que esta metodología pretende enseñar a tráves de procesos memorísticos, sin permitir un proceso más didáctico, lo que se traduce a conocimientos muy básicos, por tanto su consolidación no será sólida (Alvarado, 2014).

Con respecto a esto, el juego de roles debe dar alternativas a las diferente circustancias que sobresalen en un momento determinado, de los cuales la gente tiene o no algún conociemiento. Es fundamental, crear esos espacios en los que se generen momentos de desafíos cognitivos, debates, sugerencías, aplicación ética y creación de argumentos lógicos al mismo tiempo todos estos procesos serán interiorizados por el alumno y permitirá que la obtención de habilidades sean trasladadas no solo a la parte hipotética si no también a su actuar (Crujeiras-Pérez et al., 2020). 


\section{MÉTODO}

La presente investigación fue de tipo descriptiva no experimental mediante el paradigma epistemológico cuantitativo de cohorte transversal, la población fue tomada de los 44 estudiantes del bachillerato técnico agropecuario de la Unidad Educativa "José Benigno Iglesias", ubicada en la Provincia de Cañar, Cantón Biblián, del cual se tomó una muestra estratificada de 13 estudiantes que corresponden al tercero de bachillerato.

En primer lugar, se procedió a realizar una encuesta de 25 preguntas utilizando una escala de Likert mediante la herramienta Microsoft Forms, cuyo enlace fue enviado a través de la aplicación WhatsApp con el objetivo de indagar si los estudiantes tenían o no conocimiento de las diferentes metodologías activas las cuales se enfocaron al aula invertida y juego de roles, a más de ello, se pretendía conocer el nivel de interés que los docentes generan en sus estudiantes al momento de impartir sus asignaturas técnicas, los datos de esta encuesta se validaron con el Software SPSS, su fiabilidad se determinó por el coeficiente de correlación alfa de Cronbach con 0,851.

A más de ello, se utilizó una rúbrica de evaluación que permitió determinar diferentes criterios, verificar el avance de cada alumno en este proceso y evidenciar las acciones de los estudiantes sobre una actividad o tarea específica y como llegan a las posibles soluciones de la problemática presentada. Los criterios de evaluación fueron: 1) Participación, 2) Motivación, 3) Pensamiento reflexivo, 4) Resolución de problemas y 5) Dominio del proceso de enseñanza-aprendizaje, esta fue validada con un coeficiente de 0,748 .

Las dos metodologías implementadas se las realizó durante la hora clase que consta de 40 minutos, se envió un video previo para ser analizado, así como lecturas sobre el tema a tratarse, en clase se dividió a los estudiantes en 3 grupos de 4 personas, está fue diferente a la encuesta ya que, por razones de conectividad no todos los alumnos pudieron conectarse. A más de esto, también se pidió asignarse un rol específico y a su vez colocar un nombre a su grupo, se planteó un problema para que ellos encuentren la solución al mismo, finalmente se realizó la entrega del producto final. 


\section{RESULTADOS}

El creciente desinterés y la poca motivación de los estudiantes por las materias técnicas, son una de las dificultades muy marcadas para generar trabajo investigativo, reflexivo, crítico, analítico y por sobre todo formar personas competentes, capaces de responder a las dificultades dentro de su perfil profesional, por tanto se muestra los porcentajes del nivel de interés y estimulación que un docente provee a sus alumnos. Una vez recolectados los datos y hecho los análisis con tablas de frecuencias, se presentan los más relevantes:

\section{Tabla 1}

Conocimiento de metodologías activas, nivel de motivación y desarrollo de destrezas.

\begin{tabular}{cccccc}
\hline Ítem & & Frecuencia & Porcentaje & Porcentaje válido & $\begin{array}{c}\text { Porcentaje } \\
\text { acumulado }\end{array}$ \\
\hline $\begin{array}{c}\text { Conocimiento de aula } \\
\text { invertida }\end{array}$ & Nada & 4 & 30,8 & 30,8 & 30,8 \\
& Casi nada & 4 & 30,8 & 30,8 & 61,5 \\
& Poco & 4 & 30,8 & 30,8 & 92,3 \\
& Mucho & 1 & 7,7 & 7,7 & 100,0 \\
& Total & 13 & 100,0 & 100,0 & \\
\hline Conocimiento de juego & Nada & 1 & 7,7 & 7,7 & 7,7 \\
de roles & Casi nada & 1 & 7,7 & 7,7 & 15,4 \\
& Poco & 8 & 61,5 & 61,5 & 76,9 \\
& Mucho & 3 & 23,1 & 23,1 & 100,0 \\
Fortalecimiento en el & Total & 13 & 100,0 & 100,0 & 7,7 \\
aprendizaje con el uso & Nada & 1 & 7,7 & 7,7 & 38,5 \\
de metodologías & Poco & 4 & 30,8 & 30,8 & 100,0 \\
activas & Mucho & 8 & 61,5 & 61,5 &
\end{tabular}


Revista Arbitrada Interdisciplinaria KOINONIA

Año VI. Vol VI. N³. Edición Especial: Educación II. 2021

Hecho el depósito de Ley: FA2016000010 ISSN: $2542-3088$

FUNDACIÓN KOINONIA (F.K). Santa Ana de Coro. Venezuela.

Christian Gerardo Quinteros-Pallarozo; Nancy Marcela Cárdenas-Cordero

\begin{tabular}{cccccc}
\hline Fácil comprensión con & Poco & 4 & 30,8 & 30,8 & 30,8 \\
el uso de metodologías & Mucho & 9 & 69,2 & 69,2 & 100,0 \\
\cline { 2 - 2 } & Total & 13 & 100,0 & 100,0 & \\
\hline Metodologías & A veces & 11 & 84,6 & 84,6 & 84,6 \\
inapropiadas en & Frecuentemente & 2 & 15,4 & 15,4 & 100,0 \\
clases & Total & 13 & 100,0 & 100,0 & \\
\hline
\end{tabular}

Fuente: Encuesta.

El análisis realizado en la tabla 1, se determina que en relación al conocimiento de los estudiantes sobre las metodologías activas, se observó una distribución igualitaria del $30,8 \%$ desde nada hasta poco para el aula invertida, no así para el juego de roles, ya que, el $61,5 \%$ dice conoccer poco esta metodología, lo que quiere decir que, su uso dentro de las aulas ha sido casi nula o la aplicación de ellas es inapropiada. A más de esto, se observó que su implementación podría fortalecer el aprendizaje en un 61,5\% además de facilitar la comprensión de algunos temas que pueden ser de dificíl asimilación en un $69,2 \%$, simpre y cuando las didácticas que el docente utilice sean las adecuadas. 
Revista Arbitrada Interdisciplinaria KOINONIA

Año VI. Vol VI. N³. Edición Especial: Educación II. 2021

Hecho el depósito de Ley: FA2016000010 ISSN: 2542-3088

FUNDACIÓN KOINONIA (F.K). Santa Ana de Coro. Venezuela.

Christian Gerardo Quinteros-Pallarozo; Nancy Marcela Cárdenas-Cordero

Tabla 2.

Importancia de la formación académica en el Bachillerato Técnico por parte del estudiante.

\begin{tabular}{|c|c|c|c|c|c|}
\hline Ítem & & Frecuencia & Porcentaje & Porcentaje válido & $\begin{array}{l}\text { Porcentaje } \\
\text { acumulado }\end{array}$ \\
\hline \multirow{4}{*}{$\begin{array}{c}\text { Adquisición de } \\
\text { aprendizaje transmitido } \\
\text { por el docente en el BT }\end{array}$} & Poco & 7 & 53,8 & 53,8 & 53,8 \\
\hline & Mucho & 5 & 38,5 & 38,5 & 92,3 \\
\hline & T Demasiado & 1 & 7,7 & 7,7 & 100,0 \\
\hline & Total & 13 & 100,0 & 100,0 & \\
\hline \multirow{4}{*}{$\begin{array}{c}\text { Clases aburridas por } \\
\text { parte del docente }\end{array}$} & Nada & 3 & 23,1 & 23,1 & 23,1 \\
\hline & Casi nada & 1 & 7,7 & 7,7 & 30,8 \\
\hline & Poco & 9 & 69,2 & 69,2 & 100,0 \\
\hline & Total & 13 & 100,0 & 100,0 & \\
\hline \multirow{4}{*}{$\begin{array}{l}\text { Genera el docente } \\
\text { interés en la clase }\end{array}$} & Poco & 5 & 38,5 & 38,5 & 38,5 \\
\hline & Mucho & 7 & 53,8 & 53,8 & 92,3 \\
\hline & Demasiado & 1 & 7,7 & 7,7 & 100,0 \\
\hline & Total & 13 & 100,0 & 100,0 & \\
\hline \multirow{3}{*}{$\begin{array}{l}\text { Fortalecimiento de } \\
\text { competencias para la } \\
\text { solución de } \\
\text { problemas }\end{array}$} & Poco & 9 & 69,2 & 69,2 & 69,2 \\
\hline & Mucho & 4 & 30,8 & 30,8 & 100,0 \\
\hline & Total & 13 & 100,0 & 100,0 & \\
\hline \multirow{3}{*}{$\begin{array}{c}\text { Temática poco } \\
\text { interesante }\end{array}$} & Casi siempre & 1 & 7,7 & 7,7 & 7,7 \\
\hline & A veces & 12 & 92,3 & 92,3 & 100,0 \\
\hline & Total & 13 & 100,0 & 100,0 & \\
\hline
\end{tabular}

Fuente: Encuesta.

En la tabla 2 se evidencia que el $53,8 \%$ de los alumnos han adquirido poco aprendizaje en el Bachillerato Técnico, esto a pesar de que el $69,2 \%$ manifiesta que las clases impartidas por los docentes son poco aburridas y un $53,8 \%$ se interesan en la transmisión de conociemientos por parte del profesor, lo que no ocurre con la temática seleccionada pues un $69,2 \%$ menciona que a veces se genera dicho interés en los mismos. Esto nos 
lleva a pensar que, si bien es cierto, en las clases existe una ligera motivación, el fortalecimiento de competencias para la solución de problemas dentro de su contexto es poco. Con base a lo antes expuesto, se puede suponer que dentro de las clases no se utiliza metodologías activas para crear pensamiento reflexivo, crítico, nivel cognitivo e investigativo pues si bien es cierto, estas características ayudan a resolver las adversidades que se presentan en su quehacer diario.

\section{Tabla 3.}

Resultados de la aplicación de la rúbrica de evaluación.

\begin{tabular}{|c|c|c|c|c|c|}
\hline Ítem & & Frecuencia & Porcentaje & Porcentaje válido & $\begin{array}{l}\text { Porcentaje } \\
\text { acumulado }\end{array}$ \\
\hline \multirow[t]{3}{*}{ Participación en clase } & Regular & 4 & 33,3 & 33,3 & 33,3 \\
\hline & Bueno & 8 & 66,7 & 66,7 & 100,0 \\
\hline & Total & 12 & 100,0 & 100,0 & \\
\hline \multirow{3}{*}{$\begin{array}{l}\text { Motivación con el } \\
\text { método utilizado }\end{array}$} & Regular & 1 & 8,3 & 8,3 & 8,3 \\
\hline & Bueno & 11 & 91,7 & 91,7 & 100,0 \\
\hline & Total & 12 & 100,0 & 100,0 & \\
\hline \multirow[t]{3}{*}{ Pensamiento reflexivo } & Regular & 6 & 50,0 & 50,0 & 50,0 \\
\hline & Bueno & 6 & 50,0 & 50,0 & 100,0 \\
\hline & Total & 12 & 100,0 & 100,0 & \\
\hline \multirow{3}{*}{$\begin{array}{c}\text { Resolución de } \\
\text { problemas }\end{array}$} & Regular & 7 & 58,3 & 58,3 & 58,3 \\
\hline & Bueno & 5 & 41,7 & 41,7 & 100,0 \\
\hline & Total & 12 & 100,0 & 100,0 & \\
\hline \multirow{4}{*}{$\begin{array}{c}\text { Dominio de procesos } \\
\text { de enseñanza- } \\
\text { aprendizaje }\end{array}$} & Insuficiente & 1 & 8,3 & 8,3 & 8,3 \\
\hline & Regular & 7 & 58,3 & 58,3 & 66,7 \\
\hline & Bueno & 4 & 33,3 & 33,3 & 100,0 \\
\hline & Total & 12 & 100,0 & 100,0 & \\
\hline
\end{tabular}

Fuente: Encuesta. 
Como se observa en la tabla 3, la implementación del aula invertida combinada con el juego de roles, muestra que la participación en clases de los 12 estudiantes se incrementó en un $66,7 \%$, así como también la motivación fue mayor. En relación con el pensamiento reflexivo se determinó que hay un balance entre regular y bueno, puesto que, la falta de conocimiento del aula invertida y juego de roles, no era muy familiar para ellos, como muestra la tabla 1, por tanto, podemos determinar que, en la resolución de problemas y el dominio de procesos de enseñanza-aprendizaje fue del 58,3\% respectivamente, siendo regular para estas dos variables. Por otra parte, el principal objetivo de la investigación fue, si la implementación del aula invertida y juego de roles generaban motivación e interés en las materias técnicas de los estudiantes de Tercero de Bachillerato Producción Agropecuaria, los resultados determinaron que este si se logró alcanzar de forma satisfactoria.

\section{PROPUESTA}

Si bien es cierto que las metodologías activas permiten generar una mayor motivación, interés, desafíos cognitivos, criticidad, entre otros, la mayoría de ellas han sido utilizadas aisladamente, solo el aula invertida ha sido provado con otras didácticas, como ya se mencionó antes.

La combinación del aula invertida y el juego de roles, como estratégia para generar motivación, interés, participación y adquisición de destrezas en materias del Bachillerato Técnico, debe ser implementado como parte de la didáctica de los profesores de la figura profesional Producción Agropecuaria ya que, su falta de ejecución dentro de las aulas ha sido muy poca, lo que dará paso a que los estudiantes se vean involucrados en procesos de resolución de problemas que se puedan dar en el sector Agrícola, que va de la mano con su formación. Además, el uso de materiales que responden a la reflexión, a la construcción de conocimiento y sobre todo al desarrollo de competencias tan anheladas, permiten el vínculo entre la parte teórica con la práctica. 
Para apoyar estas metodologías activas y su implementación de forma combinada, se propone efectuar el APRAE que sus siglas representan: Analizar, preparar, resolver, actuar y evaluar.

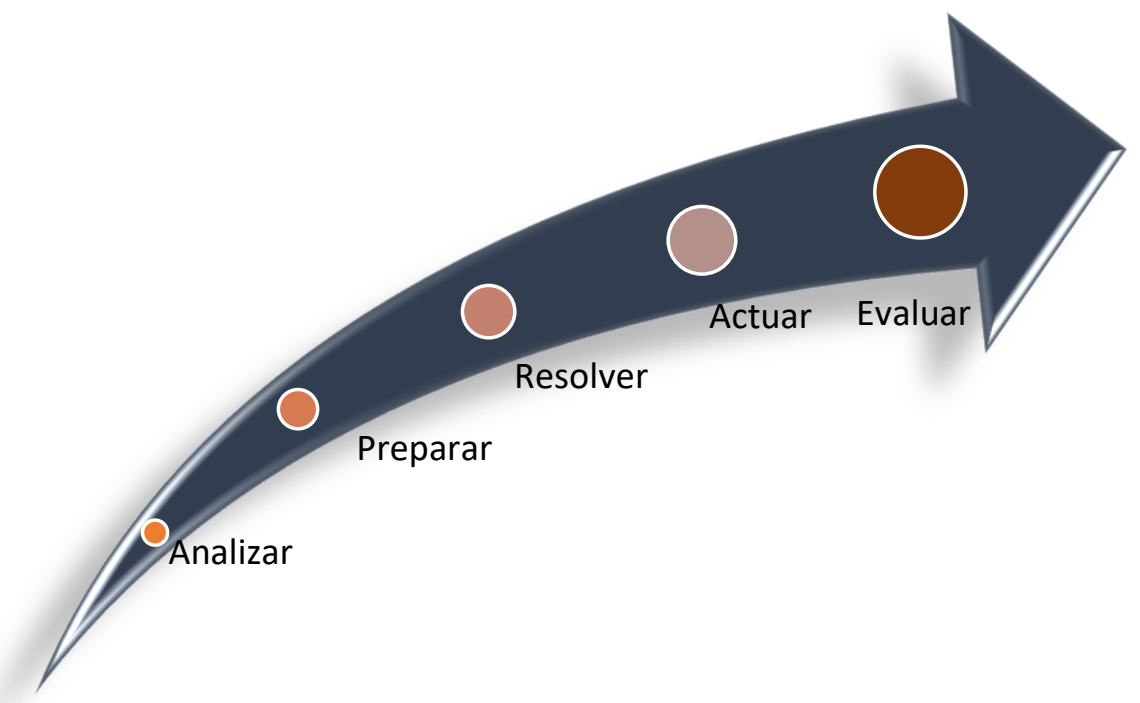

Figura 1. Etapas de la propuesta APRAE.

Elaboración: Los autores.

\section{Etapa 1}

Analizar: Con base a los temas a tratar se definirá los objetivos de aprendizaje y las competencias a desarrollar en los estudiantes con ejemplos apegados a la realidad, se recomienda analizar y desarrollar videos introductorios a la temática a tratar en clase enfocado en generar curiosidad, interés y motivación. Por otra parte, es necesario analizar el tiempo que tomará realizar cada paso propuesto, ya que, la hora clase es muy limitada y puede crear dificultades al no concluir las actividades propuestas. 


\section{Etapa 2}

Preparar: Es fundamental preparar materiales sencillos y de fácil comprensión para los alumnos y así, familiarizarse con los temas a tratar durante la hora clase. Como recomendación es ideal desarrollar una matriz en una hoja Word donde se detalle las actividades que los estudiantes realizarán durante la clase. A más de esto se debe establecer las reglas del juego, formar grupos pequeños máximo de cinco personas, proponer a los estudiantes elijan sus roles y el nombre de su grupo.

\section{Etapa 3}

Resolver: Durante el desarrollo de la clase, como primer punto importante es la realización de un pequeño sondeo mediante preguntas a los estudiantes, para verificar si los materiales entregados fueron revisados por los mismos, lo que permitirá medir el grado de comprensión del tema y resolver dudas que hayan surgido en este proceso.

\section{Etapa 4}

Actuar: Se entregará un problema a resolver en cada grupo, ellos tendrán que actuar ante este y llegar a las posibles soluciones, es importante observar que cada estudiante se involucre en el rol que eligió, también es fundamental monitorear los argumentos que se generan para resolver el problema, las diferentes opiniones y dudas que puedan surgir, mismas que serán resueltas con la guía del profesor.

\section{Etapa 5}

Evaluar: La evaluación se realizará con una rúbrica y debe hacerse durante toda la clase, de esta forma se determinará el progreso que cada estudiante adquirió, por último, se pedirá que los resultados finales de la resolución del problema planteado sean expuestos. Es necesario realizar una retroalimentación y de esta forma consolidar el conocimiento. 


\section{CONCLUSIONES}

Las metodologías activas ofrecen una gran alternativa para trabajar diferentes aspectos a ser desarrollados en los estudiantes, algunas de ellas pueden ser la creatividad, participación, motivación, reflexión, desarrollo de destrezas y desafios cognitivos, pero por sobre todo abre el camino para proporcionar las capacidades necesarias ante los problemas que surgen en los diferentes contextos. Sin embargo, no se ha evidenciado su uso en las materias del Bachillerato Técnico, generando en los estudiantes poco interés por participar en clase, la desmotivación al momento de interactuar también ha dificultado que los alumnos no adquieran las habilidades que su perfil profesional to demanda.

Por otra parte, investigaciones realizadas muestran que el aula invertida junto con otras metodologías nos brindan la oportunidad de trabajar estos aspectos, únicamente el juego de roles no registra trabajo con didácticas diferentes pero, también nos ayudan a fortalecer los mismos. Es por esto que, la implemetación de manera unánime nos provee un abaníco de oportunidades para operar de manera dinámica dentro del aula. Seguido de esto, es imporatnte que se analice las estrategias para trabajar en el aula, por mencionar algunos son: los objetivos que se desean alcanzar en cada sesión, la preparación de los materiales, temas de interés y coordinación del tiempo.

Con los resultados obtenidos podemos ver que el porcentaje de los estudiantes en cuanto a la participación subió en un $66,7 \%$, así como su motivación en un $91,7 \%$ lo que quiere decir que, la combinación de estas dos metodologías nos permite generar interés ante una temática propuesta. Por otra parte, el pensamiento reflexivo no tuvo un gran incremento ya que, solo el $50 \%$ de estudiantes pudieron dar un argumento acorde al problema propuesto, de la misma forma la resolución de este, el domonio de aprendizajes fue regular, pues el poco conocimiento de estas metodologías dificulta el trabajo investigativo que los alumnos deben realizar previo a su implementación, por lo que es indispensable que se vaya generando una cultura dentro de las clases impartidas sobre cada una de las metodologías activas. 
Si bien es cierto, la implementación de las dos pedagogías fortalece los conocimientos de las diferentes asignaturas del Bachillerato Técnico, incrementar la participación y motivación, durante su proceso puede presentar algunas dificultades, ya sea por el corto tiempo que se tiene o que los estudiantes no revisen los materiales enviados para su estudio. Al mismo tiempo, se dificultará el fortalecimiento del conocimeinto y la resolución de problemas.

Teniendo en cuenta todo lo antes mencionado, se puede concluir que, la implementación conjunta de las metodologías ayudan a alcanzar las competencias necesarias que los estudiantes así lo demandan ya que, si se creó interés en los estudiante frente a los temas planteados. De la misma manera, es esencial realizar ciertos ajustes a las dos metodologías puesto que, su ejecución son similares, por lo que se propone el APRAE como ayuda a la fusión de estas dos técnicas, de esta manera se logrará articular cada uno de los criterios que se desean evaluar durante el proceso de enseñanza-aprendizaje.

\section{FINANCIAMIENTO}

No monetario.

\section{AGRADECIMIENTO}

A la Jefatura de Posgrados de la Universidad Católica de Cuenca por permitir el desarrollo y fomento de la investigación.

\section{REFERENCIAS CONSULTADAS}

Alvarado, C. L. (2014). Diseño de un Manual Didáctico con el Ciclo de Aprendizaje en el Módulo de Manejo Sanitario y Fitosanitario de Especies Animales y Vegetales para los Estudiantes del Primer Año de Bachillerato de la Especialización de Producciones Agropecuarias del Colegio [Design of a Didactic Manual with the Learning Cycle in the Sanitary and Phytosanitary Management Module of Animal and Vegetable Species for the Students of the First Year of Baccalaureate of the Specialization of Agricultural Productions of the College]. https://n9.cl/o5xr0 
Revista Arbitrada Interdisciplinaria KOINONIA

Año VI. Vol VI. N³. Edición Especial: Educación II. 2021

Hecho el depósito de Ley: FA2016000010

ISSN: 2542-3088

FUNDACIÓN KOINONIA (F.K). Santa Ana de Coro. Venezuela.

Christian Gerardo Quinteros-Pallarozo; Nancy Marcela Cárdenas-Cordero

Batistello, P., \& Cybis-Pereira, A. (2019). El aprendizaje basado en competencias y metodologías activas: aplicando la gamificación [Competency-based learning and active methodologies: applying gamification]. Arquitectura y Urbanismo, $X L(2), 31$ 42.

Cantuña-Avila, A. A., \& Cañar-Tapia, C. E. (2020). Systematic review of the flipped classroom in Ecuador: approach to the state of the art. Estudios pedagógicos (Valdivia), 46(3), 45-58. https://dx.doi.org/10.4067/S0718-07052020000300045

Castro, M. S., Paz, M. L., \& Cela, E. M. (2020). Aprendiendo a enseñar en tiempos de pandemia COVID-19: nuestra experiencia en una universidad pública de Argentina [Learning to teach in times of the COVID-19 pandemic: our experience at Universidad de Buenos Aires]. Revista Digital de Investigación en Docencia Universitaria, 14(2), e1271. https://dx.doi.org/10.19083/ridu.2020.1271

Crujeiras-Pérez, B., Martín-Gamez, C., Díaz-Moreno, N., \& Fernández-Oliveras, A. (2020). Trabajar la argumentación a través de un juego de rol : ¿ debemos instalar el cementerio nuclear? [Learning argumentation through role-playing: should we install a nuclear deposit?]. Ensenanza de Las Ciencias, 3(38), 125-142. https://doi.org/10.5565/rev/ensciencias.2888

Escudero-Nahón, A., \& Mercado-López, E. P. (2019). Uso del análisis de aprendizajes en el aula invertida : una revisión sistemática [Use of learning analytics in the flipped classroom: a systematic review]. Apertura, 11, 72-85. https://doi.org/10.32870/Ap.v11n2.1546

España-Ramos, E., Rueda-Serón, J. A., \& Blanco-López, Á. (2013). Juegos de rol sobre el calentamiento global. Actividades de enseñanza realizadas por estudiantes de ciencias del Máster en Profesorado de Secundaria [Role-play on global warming. Teaching activities designed by students in preservice science teachertrain]. Revista Eureka Sobre Enseñanza y Divulgación de Las Ciencias, 10, 763-779.

Gaete-Quezada, R. A. (2011). El juego de roles como estrategia de evaluación de aprendizajes universitarios [Role play as a strategy for evaluating university learning]. Educacion y Educadores, 14(2), 289-307.

Gorbaneff, Y. (2003). Inglaterra y Holanda en Asia Sudoriental: Un caso - Juego de roles [England and Holland in Southeast Asia: A case - Role play]. Innovar, 1(22), 173178. 
Han, H., \& Røkenes, F. M. (2020). Flipped Classroom in Teacher Education : A Scoping Review. Frontiers in Education, 5. https://doi.org/10.3389/feduc.2020.601593

Hernández-Suárez, C. A., Prada-Nuñez, R., \& Gamboa-Suárez, A. A. (2020). Pre-service teacher education: active scenarios from a flipped classroom a perspective. Formación universitaria, 13(5), 213222. https://dx.doi.org/10.4067/S0718-50062020000500213

Islas-Torres, C., \& Carranza-Alcántar, M. del R. (2020). Análisis de contenido de una experiencia formativa a través de aula invertida [Content analysis of a training experience through flipped classroom]. 61, 33-18. https://doi.org/https://doi.org/10.35575/rvucn.n61a2

Konopka, C., Adaime, M. \& Mosele, P. (2015) Active Teaching and Learning Methodologies: Some Considerations. Creative Education, 6, 1536-1545. http://dx.doi.org/10.4236/ce.2015.614154

Ley orgánica de educación intercultural LOEI. Recuperado desde https://n9.cl/go3a

Medina-Díaz, M. del R., \& Verdejo-Carrión, A. (2020). Validez y confiabilidad en la evaluación del aprendizaje mediante las metodologías activas Validez y confiabilidad en la evaluación del aprendizaje mediante las metodologías activas [Validity and reliability in the evaluation of 1learning through active me]. Alteridad, 15(2). https://doi.org/10.17163/alt.v15n2.2020.10

Muntaner-Guasp, J. J., Pinya Medina, C., \& Mut Amengual, B. (2020). El impacto de las metodologías activas en los resultados académicos [The impact of active methodologies on academic results]. Profesorado, Revista de Currículum y Formación Del Profesorado, 24(1), 96-114. https://doi.org/10.30827/profesorado.v24i1.8846

Pons-García, L., \& Sonsoles-de Soto-García, I. (2020). Evaluación de una propuesta de aprendizaje basado en juegos de rol llevada a cabo en la asignatura de Cultura Científica de Bachillerato [Evaluation of a learning proposal based on role-playing games carried out in the subject of High School Scientific Cultural]. Revista de Estudios y Experiencias En Educación, 19(39), 123-144. https://doi.org/10.21703/rexe.20201939pons7 
Rodriguez, A. B., Ramirez, L. J., \& Fernández, W. (2017). Metodologías activas para alcanzar el comprender [Active methodologies to achieve understanding]. Formacion Universitaria, 10(1), 79-88. https://doi.org/10.4067/S0718$\underline{50062017000100009}$

Silva, J., \& Maturana, D. (2017). A proposal of a Model for the introduction of active methodologies in Higher Education [Una propuesta de Modelo para la introducción de metodologías activas en la Educación Superior]. Innovación Educativa, 17(73), 117-131.

Suárez-Cretton, X., Castro-Méndez, N., \& Muñoz-Vilches, C. (2020). Use of Role Playing With Video Recording to Develop Interviewing Skills in Students of Psychology. Revista Electrónica Educare, 24(2), 1-18. https://doi.org/10.15359/ree.24-2.2

Vidal, M., Rivera, N., Nolla, N., Morales, I. R., \& Vialart, M. R. (2016). Aula invertida, nueva estrategia didáctica [The flipped classroom , a new didactic strategy]. Educación Médica Superior, 30(3), 678-688. 